\title{
A blow-up result for a system of coupled viscoelastic equations with arbitrary positive initial energy
}

\section{Qian $\mathrm{Li}^{1 *}$ (1)}

"Correspondence:

liqian206@outlook.com

1 Department of Mathematics,

Changzhi University, Changzhi,

Shanxi 046000, China

\section{Springer}

\begin{abstract}
This article is devoted to a study of the blow-up result for a system of coupled viscoelastic wave equations. By establishing a new auxiliary function and using the reduction to absurdity method, we obtain some sufficient conditions on initial data such that the solution blows up in finite time at arbitrarily high initial energy. This work generalizes and improves earlier results in the literature.
\end{abstract}

Keywords: Viscoelastic wave equation; Relaxation function; Blow up

\section{Introduction}

In this article, we investigate the blow-up property of the coupled viscoelastic wave equations of the form

$$
\left\{\begin{array}{l}
\left|u_{t}\right|^{\rho} u_{t t}-\Delta u+\int_{0}^{t} g_{1}(t-\tau) \Delta u(\tau) d \tau+\left|u_{t}\right|^{m-2} u_{t}=f_{1}(u, v), \quad x \in \Omega, t>0, \\
\left|v_{t}\right|^{\rho} v_{t t}-\Delta v+\int_{0}^{t} g_{2}(t-\tau) \Delta v(\tau) d \tau+\left|v_{t}\right|^{r-2} v_{t}=f_{2}(u, v), \quad x \in \Omega, t>0, \\
u(x, t)=v(x, t)=0, \quad x \in \partial \Omega, t>0, \\
u(x, 0)=u_{0}(x), \quad u_{t}(x, 0)=u_{1}(x), \quad x \in \Omega, \\
v(x, 0)=v_{0}(x), \quad v_{t}(x, 0)=v_{1}(x), \quad x \in \Omega .
\end{array}\right.
$$

Here $\Omega$ is a bounded domain of $R^{n}$ with a smooth boundary $\partial \Omega . \rho>0, g_{1}$ and $g_{2}$ are the kernel of memory terms, the nonlinear terms $f_{1}$ and $f_{2}$ will be specified later. The problem of (1.1) has been considered by many mathematics researchers and results in connection with blow-up and decay have been extensively established.

For single viscoelastic wave equation, Messaoudi [11] discussed the following equation:

$$
\left\{\begin{array}{l}
u_{t t}-\Delta u+\int_{0}^{t} g(t-\tau) \Delta u(\tau) d \tau+u_{t}\left|u_{t}\right|^{m-2}=u|u|^{p-2}, \quad x \in \Omega, t>0, \\
u(x, t)=0, \quad x \in \partial \Omega, t \geq 0, \\
u(x, 0)=u_{0}(x), \quad u_{t}(x, 0)=u_{1}(x), \quad x \in \Omega,
\end{array}\right.
$$

(c) The Author(s) 2021. This article is licensed under a Creative Commons Attribution 4.0 International License, which permits use sharing, adaptation, distribution and reproduction in any medium or format, as long as you give appropriate credit to the original author(s) and the source, provide a link to the Creative Commons licence, and indicate if changes were made. The images or other third party material in this article are included in the article's Creative Commons licence, unless indicated otherwise in a credit line to the material. If material is not included in the article's Creative Commons licence and your intended use is not permitted by statutory regulation or exceeds the permitted use, you will need to obtain permission directly from the copyright holder. To view a copy of this licence, visit http://creativecommons.org/licenses/by/4.0/. 
where $m \geq 1, p \geq 2, \Omega$ is a bounded domain. The author studied the interaction between the weak damping term $u_{t}\left|u_{t}\right|^{m-2}$ and the nonlinear source term $u|u|^{p-2}$, which was first considered by Levine $[9,10]$ when $m=1$, and found, under suitable conditions on $g$ and initial data, that the solutions exist globally for any initial data if $m \geq p$ and blow up in finite time with negative initial energy if $p>m$. This blow-up result has been pushed by the same author in [12], to certain solutions with positive initial energy. Recently, Song [19] proved, by using the reduction to absurdity method, that the solutions of Eq. (1.2) blow up in finite time with the initial data have arbitrarily high initial energy. In the case when the nonlinear damping term $u_{t}\left|u_{t}\right|^{m-2}$ is replaced by the strong damping term $-\Delta u_{t}$ in Eq. (1.2), Song and Zhong [21] showed, by using the potential well theory introduced by Payne and Sattinger [16], that a blow-up result for solutions with positive initial energy. Later, Song and Xue [20] improved the blow-up result in which the initial data have arbitrarily high initial energy. In [22], Xu and Lian studied a nonlinear wave equation with weak and strong damping terms and logarithmic source term, they established the local existence of weak solution, showed the global existence, energy decay in the framework of potential well and obtained the blow-up of the solution with sub-critical initial energy. Furthermore, they in parallel extend all the conclusions for the sub-critical case to the critical case by scaling technique. Besides, a high energy infinite time blow-up result is established. Within a similar potential well framework, the semilinear pseudo parabolic equation [24] and parabolic system [23] were discussed in depth.

In the same direction, Song [18] discussed the following initial-boundary value problem:

$$
\left|u_{t}\right|^{\rho} u_{t t}-\Delta u+\int_{0}^{t} g(t-\tau) \Delta u(\tau) d \tau+u_{t}\left|u_{t}\right|^{m-2}=u|u|^{p-2}, \quad x \in \Omega, t>0,
$$

where $\Omega$ is a bounded domain and $\rho>0, m \geq 1, p>2$. By modifying the method used in Messaoudi [12], the author proved that the solution blows up in finite time with initial data have positive initial energy. Recently, He and Song [8] pushed the blow-up result to certain solutions with arbitrary positive initial energy. When the nonlinear damping term $u_{t}\left|u_{t}\right|^{m-2}$ is substituted by the strong damping term $-\Delta u_{t}$, Hao et al. [7], inspired by the method used in Song [19], proved that solutions with negative initial energy as well as positive initial energy blow-up in finite time provided $p>\rho+2$, and obtained, by using the perturbed energy functional technique, that solutions exist globally for any initial data provided $p \leq \rho+2$.

For a blow-up result in systems of hyperbolic equations, the coupled system

$$
\left\{\begin{array}{l}
u_{t t}-\Delta u+u_{t}\left|u_{t}\right|^{m-1}=f_{1}(u, v), \quad x \in \Omega, t>0, \\
v_{t t}-\Delta v+v_{t}\left|v_{t}\right|^{r-1}=f_{2}(u, v), \quad x \in \Omega, t>0, \\
u(x, t)=v(x, t)=0, \quad x \in \partial \Omega, t>0, \\
u(x, 0)=u_{0}(x), \quad u_{t}(x, 0)=u_{1}(x), \quad x \in \Omega, \\
v(x, 0)=v_{0}(x), \quad v_{t}(x, 0)=v_{1}(x), \quad x \in \Omega,
\end{array}\right.
$$

was considered by Agre and Rammaha [2], where $m, r \geq 1$ and $\Omega$ is a bounded domain with smooth boundary. The authors found, by using the same method as in [4], that any weak solution blows up in finite time with negative initial energy. Furthermore, Said-Houari [17] extended this blow-up result to positive initial energy. 
In the presence of the memory term, Han and Wang [5] considered the following system of viscoelastic equations:

$$
\left\{\begin{array}{l}
u_{t t}-\Delta u+\int_{0}^{t} g(t-\tau) \Delta u(\tau) d \tau+u_{t}\left|u_{t}\right|^{m-1}=f_{1}(u, v), \quad x \in \Omega, t>0, \\
v_{t t}-\Delta v+\int_{0}^{t} h(t-\tau) \Delta v(\tau) d \tau+v_{t}\left|v_{t}\right|^{r-1}=f_{2}(u, v), \quad x \in \Omega, t>0 \\
u(x, t)=v(x, t)=0, \quad x \in \partial \Omega, t>0, \\
u(x, 0)=u_{0}(x), \quad u_{t}(x, 0)=u_{1}(x), \quad x \in \Omega, \\
v(x, 0)=v_{0}(x), \quad v_{t}(x, 0)=v_{1}(x), \quad x \in \Omega,
\end{array}\right.
$$

They obtained the local existence, global existence, uniqueness and a blow-up result for certain solutions with negative initial energy. In [13], Messaoudi and Said-Houari extended this blow-up result to certain solutions with positive initial energy. Later, Zhao and Wang [1] proved the finite time blow-up of solutions whose initial data have arbitrarily high initial energy. In the same nature, Mustafa [14] considered a coupled system of nonlinear viscoelastic equations, he proved the well-posedness and established a generalized stability result for this system. More relevant knowledge we refer the reader to the literature [15].

As far as we know, the problem of the blow-up phenomenon for system (1.1) with arbitrary positive initial energy has not been considered. Our aim in this paper is to extend the research method for the blow-up phenomena used in [8] to the couple viscoelastic wave system (1.1), while we should handle the additional difficulty caused by damping term, viscoelastic term and source term. In order to overcome the difficulty, we construct a suitable auxiliary functions $\left(u, \frac{u_{t}\left|u_{t}\right|^{\rho}}{\rho+1}\right)+\left(v, \frac{v_{t}\left|\nu_{t}\right|^{\rho}}{\rho+1}\right)-\left(\frac{1}{\varepsilon_{1}^{*}}\right)^{\frac{\gamma^{*}}{\gamma^{*}-1}} \frac{\gamma-1}{\gamma} E(t)$ and combine the reduction to absurdity method to derive contradiction, namely, we find suitable conditions on initial data such that the solution of (1.1) blows up in finite time at arbitrary high initial energy level.

This article is organized as follows. In Sect. 2, we present some material needed for our work. Section 3 is devoted to the blow-up result.

\section{Preliminaries}

In this section, we give some material needed for our work. Firstly, let us make the following assumptions.

(A1) $g_{i}: R_{+} \rightarrow R_{+}$(for $\left.i=1,2\right)$ are non-increasing differentiable functions satisfying

$$
g_{i}(0)>0, \quad 1-\int_{0}^{\infty} g_{i}(\tau) d \tau=l_{i}>0 .
$$

(A2) For nonlinear terms, we assume that

$$
\begin{aligned}
& 0<\rho<+\infty, n=1,2, \quad 0<\rho \leq \frac{2}{n-2}, \quad n \geq 3, \\
& 2 \leq m, r<+\infty, n=1,2, \quad 2 \leq m, r \leq \frac{2 n}{n-2}, \quad n \geq 3 .
\end{aligned}
$$

(A3) For the functions $f_{1}$ and $f_{2}$, we note that

$$
f_{1}(u, v)=\left[a|u+v|^{2(p+1)}(u+v)+b|u|^{p} u|v|^{p+2}\right],
$$




$$
f_{2}(u, v)=\left[a|u+v|^{2(p+1)}(u+v)+b|v|^{p} v|u|^{p+2}\right], \quad a, b>0,
$$

where

$$
-1<p<+\infty, n=1,2, \quad-1<p \leq \frac{3-n}{n-2}, \quad n \geq 3 .
$$

It is easy to verify that

$$
u f_{1}(u, v)+v f_{2}(u, v)=2(p+2) F(u, v), \quad \forall(u, v) \in \mathbb{R}^{2},
$$

where

$$
F(u, v)=\frac{1}{2(p+2)}\left[a|u+v|^{2(p+2)}+2 b|u v|^{p+2}\right] .
$$

At present, we state the following local existence theorem which can be proved by combining the arguments in $[3,6]$. Here we omit the proof.

Theorem 2.1 Suppose that (A1), (A2), (A3) hold and $u_{0}, v_{0} \in H_{0}^{1}(\Omega), u_{1}, v_{1} \in L^{2}(\Omega)$ are given, then system (1.1) possesses a unique local solution $(u, v)$ such that

$$
\begin{aligned}
& (u, v) \in C\left([0, T] ; H_{0}^{1}(\Omega)\right) \times C\left([0, T] ; H_{0}^{1}(\Omega)\right), \\
& \left(u_{t}, v_{t}\right) \in C\left([0, T] ; L^{2}(\Omega)\right) \cap L^{m}(\Omega \times[0, T]) \times C\left([0, T] ; L^{2}(\Omega)\right) \cap L^{r}(\Omega \times[0, T]),
\end{aligned}
$$

for the maximum existence time $T>0$, where $T \in(0, \infty]$.

The energy of the system (1.1) is

$$
\begin{aligned}
E(t)= & \frac{1}{\rho+2}\left(\left\|u_{t}\right\|_{\rho+2}^{\rho+2}+\left\|v_{t}\right\|_{\rho+2}^{\rho+2}\right)+\frac{1}{2}\left(1-\int_{0}^{t} g_{1}(\tau) d \tau\right)\|\nabla u\|_{2}^{2}-\int_{\Omega} F(u, v) d x \\
& +\frac{1}{2}\left[\left(g_{1} \circ \nabla u\right)+\left(g_{2} \circ \nabla v\right)\right]+\frac{1}{2}\left(1-\int_{0}^{t} g_{2}(\tau) d \tau\right)\|\nabla v\|_{2}^{2},
\end{aligned}
$$

where

$$
(g \circ \nabla v)(t)=\int_{0}^{t} g(t-\tau)\|v(t)-v(\tau)\|_{2}^{2} d \tau .
$$

Lemma 2.2 Assume (A1) holds, and let $(u, v)$ be a solution of system (1.1), then $E(t)$ is non-increasing, namely

$$
\frac{d E(t)}{d t} \leq-\left\|u_{t}\right\|_{m}^{m}-\left\|v_{t}\right\|_{r}^{r} \leq 0, \quad \forall t \geq 0
$$

Proof Multiplying the first two equations in system (1.1) by $u_{t}, v_{t}$, respectively, and then integrating over $\Omega$, we get

$$
\begin{aligned}
& \frac{d}{d t}\left[\frac{1}{\rho+2}\left(\left\|u_{t}\right\|_{\rho+2}^{\rho+2}+\left\|v_{t}\right\|_{\rho+2}^{\rho+2}\right)+\frac{1}{2}\left(\|\nabla u\|_{2}^{2}+\|\nabla v\|_{2}^{2}\right)-\int_{\Omega} F(u, v) d x\right] \\
& \quad=-\left\|u_{t}\right\|_{m}^{m}-\left\|v_{t}\right\|_{r}^{r}+\int_{0}^{t} g_{1}(t-\tau) \int_{\Omega} \nabla u_{t}(t) \cdot \nabla u(\tau) d x d \tau
\end{aligned}
$$




$$
+\int_{0}^{t} g_{2}(t-\tau) \int_{\Omega} \nabla v_{t}(t) \cdot \nabla v(\tau) d x d \tau
$$

where

$$
\begin{aligned}
\int_{0}^{t} g_{1}(t-\tau) \int_{\Omega} \nabla u_{t}(t) \cdot \nabla u(\tau) d x d \tau \\
=\int_{0}^{t} g_{1}(t-\tau) \int_{\Omega} \nabla u_{t}(t) \cdot[\nabla u(\tau)-\nabla u(t)] d x d \tau \\
\quad+\int_{0}^{t} g_{1}(t-\tau) \int_{\Omega} \nabla u_{t}(t) \cdot \nabla u(t) d x d \tau \\
=-\frac{1}{2} \int_{0}^{t} g_{1}(t-\tau)\left(\frac{d}{d t} \int_{\Omega}|\nabla u(\tau)-\nabla u(t)|^{2} d x\right) d \tau \\
\quad+\int_{0}^{t} g_{1}(\tau)\left(\frac{d}{d t} \frac{1}{2} \int_{\Omega}|\nabla u(t)|^{2} d x\right) d \tau \\
=-\frac{1}{2} \frac{d}{d t}\left[\int_{0}^{t} g_{1}(t-\tau) \int_{\Omega}|\nabla u(\tau)-\nabla u(t)|^{2} d x d \tau\right] \\
\quad+\frac{1}{2} \frac{d}{d t}\left[\int_{0}^{t} g_{1}(\tau) \int_{\Omega}|\nabla u(t)|^{2} d x d \tau\right] \\
\quad+\frac{1}{2} \int_{0}^{t} g_{1}^{\prime}(t-\tau) \int_{\Omega}|\nabla u(\tau)-\nabla u(t)|^{2} d x d \tau-\frac{1}{2} g_{1}(t) \int_{\Omega}|\nabla u(t)|^{2} d x .
\end{aligned}
$$

Similarly

$$
\begin{aligned}
\int_{0}^{t} g_{2}(t-\tau) \int_{\Omega} \nabla v_{t}(t) \cdot \nabla v(\tau) d x d \tau \\
=-\frac{1}{2} \frac{d}{d t}\left[\int_{0}^{t} g_{2}(t-\tau) \int_{\Omega}|\nabla v(\tau)-\nabla v(t)|^{2} d x d \tau\right] \\
\quad+\frac{1}{2} \frac{d}{d t}\left[\int_{0}^{t} g_{2}(\tau) \int_{\Omega}|\nabla v(t)|^{2} d x d \tau\right] \\
+\frac{1}{2} \int_{0}^{t} g_{2}^{\prime}(t-\tau) \int_{\Omega}|\nabla v(\tau)-\nabla v(t)|^{2} d x d \tau-\frac{1}{2} g_{2}(t) \int_{\Omega}|\nabla v(t)|^{2} d x .
\end{aligned}
$$

By inserting (2.3) and (2.4) into (2.2), and combining (A1), we can obtain

$$
\begin{aligned}
\frac{d}{d t}[ & \frac{1}{\rho+2}\left(\left\|u_{t}\right\|_{\rho+2}^{\rho+2}+\left\|v_{t}\right\|_{\rho+2}^{\rho+2}\right)+\frac{1}{2}\left(1-\int_{0}^{t} g_{1}(\tau) d \tau\right)\|\nabla u\|_{2}^{2} \\
& +\left(1-\int_{0}^{t} g_{2}(\tau) d \tau\right)\|\nabla v\|_{2}^{2} \\
& \left.+\frac{1}{2}\left[\left(g_{1} \circ \nabla u\right)+\left(g_{2} \circ \nabla v\right)\right]-\int_{\Omega} F(u, v) d x\right] \\
= & -\left\|u_{t}\right\|_{m}^{m}-\left\|v_{t}\right\|_{r}^{r}+\frac{1}{2}\left[\left(g_{1}^{\prime} \circ \nabla u\right)+\left(g_{2}^{\prime} \circ \nabla v\right)\right]-\frac{1}{2} g_{1}(t)\|\nabla u\|_{2}^{2}-\frac{1}{2} g_{2}(t)\|\nabla v\|_{2}^{2},
\end{aligned}
$$


namely

$$
\begin{aligned}
E^{\prime}(t) & =-\left\|u_{t}\right\|_{m}^{m}-\left\|v_{t}\right\|_{r}^{r}+\frac{1}{2}\left[\left(g_{1}^{\prime} \circ \nabla u\right)+\left(g_{2}^{\prime} \circ \nabla v\right)\right] \frac{1}{2} g_{1}(t)\|\nabla u\|_{2}^{2}-\frac{1}{2} g_{2}(t)\|\nabla v\|_{2}^{2} \\
& \leq-\left\|u_{t}\right\|_{m}^{m}-\left\|v_{t}\right\|_{r}^{r} \leq 0 .
\end{aligned}
$$

Lemma 2.3 ([1], Lemma 2) There exist two positive constants $c_{0}$ and $c_{1}$ such that

$$
\frac{c_{0}}{2(p+2)}\left(|u|^{2(p+2)}+|v|^{2(p+2)}\right) \leq F(u, v) \leq \frac{c_{1}}{2(p+2)}\left(|u|^{2(p+2)}+|v|^{2(p+2)}\right) .
$$

Lemma 2.4 Assume $0<\pi<\alpha<\gamma$ hold, then we have the following inequality:

$$
\|u\|_{\alpha}^{\alpha} \leq\|u\|_{\pi}^{\pi}+\|u\|_{\gamma}^{\gamma}
$$

Proof (1) When $|u|>1$, then $\int_{\Omega}|u|^{\alpha} d x \leq \int_{\Omega}|u|^{\gamma} d x \leq \int_{\Omega}|u|^{\pi} d x+\int_{\Omega}|u|^{\gamma} d x$ is ture.

(2) When $|u| \leq 1$, then $\int_{\Omega}|u|^{\alpha} d x \leq \int_{\Omega}|u|^{\pi} d x \leq \int_{\Omega}|u|^{\pi} d x+\int_{\Omega}|u|^{\gamma} d x$ is ture, where $|\cdot|$ represents the absolute value.

Lemma 2.5 Assume $0<\varrho<\varsigma$ hold, then we have the following inequality:

$$
\|u\|_{\zeta}^{\varrho}<\|u\|_{S}^{\varsigma}+1
$$

Proof (1) Assume $\|u\|_{\varsigma}>1$ hold, then $\|u\|_{\zeta}^{\varrho}<\|u\|_{\zeta}^{\varsigma}<\|u\|_{\zeta}^{\varsigma}+1$ is ture.

(2) Assume $\|u\|_{\varsigma} \leq 1$ hold, then $\|u\|_{\zeta}^{\varrho}<\|u\|_{\varsigma}^{\varsigma}+1$ is ture.

In order to obtain our main result, we need the following lemma which presents the same one of (He and Song [8] Lemma 2.2) with suitable modification.

Lemma 2.6 ([8], Lemma 2.2) Assume that $2<\rho+2<\min \{m, r\}$ and $\max \{m, r\}<2(p+2)$. Assume further that (A1)-(A3) hold and $g_{i}(i=1,2)$ satisfying (3.1). If there exists a number $t_{0} \geq 0$ such that $E\left(t_{0}\right)<0$, then the solution of the system (1.1) blows up in finite time.

\section{Blow-up result}

In this section, we discuss the blow-up phenomenon.

Theorem 3.1 Assume the conditions (A1)-(A3) and $2<\rho+2<\min \{m, r\}, \max \{m, r\}<$ $2(p+2)$ hold, and $g_{i}$ satisfies the condition

$$
\max \left\{\int_{0}^{\infty} g_{1}(\tau) d \tau, \int_{0}^{\infty} g_{2}(\tau) d \tau\right\}<\frac{p+1}{p+1+\frac{1}{4(p+2)}}
$$

for $i=1,2$. Let $(u, v)$ be the solution of system (1.1), satisfying

$$
\int_{\Omega} u(0) \frac{u_{t}(0)\left|u_{t}(0)\right|^{\rho}}{\rho+1} d x+\int_{\Omega} v(0) \frac{v_{t}(0)\left|v_{t}(0)\right|^{\rho}}{\rho+1} d x>M E(0)>0
$$


then $(u, v)$ blows up in finite time, where

$$
M=\left(\frac{1}{\varepsilon_{1}^{*}}\right)^{\frac{\gamma^{*}}{\gamma^{*}-1}} \frac{\gamma-1}{\gamma}
$$

$\gamma=\max \{m, r\}, \gamma^{*}=\min \{m, r\}, \varepsilon_{1}^{*}>0$ is a constant such that $\left(\frac{1}{\varepsilon_{1}}\right)^{\frac{\gamma^{*}}{\gamma^{*}-1}} \frac{\gamma-1}{\gamma} \geq \frac{2(p+2)(1-\varepsilon)}{\beta}, \varepsilon \in$ $(0,1)$ is a small enough constant such that

$$
\begin{aligned}
& \kappa_{1}(\varepsilon)=((p+2)(1-\varepsilon)-1) l_{1}-\frac{1}{4(p+2)(1-\varepsilon)}\left(1-l_{1}\right)>0, \\
& \kappa_{2}(\varepsilon)=((p+2)(1-\varepsilon)-1) l_{2}-\frac{1}{4(p+2)(1-\varepsilon)}\left(1-l_{2}\right)>0, \\
& \alpha=\min \left\{\kappa_{1}(\varepsilon) \lambda-\frac{\varepsilon_{1}^{m}}{m}, \kappa_{2}(\varepsilon) \lambda-\frac{\varepsilon_{1}^{r}}{r}, c_{0} \varepsilon-\frac{\varepsilon_{1}^{m}}{m}, c_{0} \varepsilon-\frac{\varepsilon_{1}^{r}}{r}\right\}, \\
& \beta=\min \left\{\frac{1}{\rho+1}+\frac{2(p+2)(1-\varepsilon)}{\rho+2}, \alpha\right\},
\end{aligned}
$$

and $\lambda$ is the first eigenvalue of $-\Delta$.

Proof Suppose that $(u, v)$ is a global solution of system (1.1). Multiplying the first two equations of system (1.1) by $u$ and $v$, respectively, and integrating over $\Omega$, we obtain

$$
\begin{aligned}
& \left(\left|u_{t}\right|^{\rho} u_{t t}, u\right)+\|\nabla u\|_{2}^{2}+\int_{\Omega} \int_{0}^{t} g_{1}(t-\tau) \Delta u(\tau) d \tau u(t) d x+\int_{\Omega} u u_{t}\left|u_{t}\right|^{m-2} d x \\
& =\int_{\Omega} u f_{1}(u, v) d x, \\
& \left(\left|v_{t}\right|^{\rho} v_{t t}, v\right)+\|\nabla v\|_{2}^{2}+\int_{\Omega} \int_{0}^{t} g_{2}(t-\tau) \Delta v(\tau) d \tau v(t) d x+\int_{\Omega} v v_{t}\left|v_{t}\right|^{m-2} d x \\
& =\int_{\Omega} v f_{2}(u, v) d x .
\end{aligned}
$$

Taking the derivative of $\left(u, \frac{u_{t}\left|u_{t}\right|^{\rho}}{\rho+1}\right)$ and $\left(v, \frac{v_{t}\left|v_{t}\right|^{\rho}}{\rho+1}\right)$, respectively, and combining (3.3) and (3.4), we have

$$
\begin{aligned}
\frac{d}{d t}\left(u, \frac{u_{t}\left|u_{t}\right|^{\rho}}{\rho+1}\right)= & \frac{1}{\rho+1}\left\|u_{t}(t)\right\|_{\rho+2}^{\rho+2}-\|\nabla u\|_{2}^{2}+\int_{0}^{t} g_{1}(t-\tau) \int_{\Omega} \nabla u(\tau) \nabla u(t) d x d \tau \\
& -\int_{\Omega} u u_{t}\left|u_{t}\right|^{m-2} d x+\int_{\Omega} u f_{1}(u, v) d x, \\
\frac{d}{d t}\left(v, \frac{v_{t}\left|v_{t}\right|^{\rho}}{\rho+1}\right)= & \frac{1}{\rho+1}\left\|v_{t}(t)\right\|_{\rho+2}^{\rho+2}-\|\nabla v\|_{2}^{2}+\int_{0}^{t} g_{2}(t-\tau) \int_{\Omega} \nabla v(\tau) \nabla v(t) d x d \tau \\
& -\int_{\Omega} v v_{t}\left|v_{t}\right|^{r-2} d x+\int_{\Omega} v f_{2}(u, v) d x .
\end{aligned}
$$

For the third term on the right side of (3.5), we get

$$
\int_{0}^{t} g_{1}(t-\tau) \int_{\Omega} \nabla u(\tau) \nabla u(t) d x d \tau=\int_{0}^{t} g_{1}(t-\tau) \int_{\Omega} \nabla u(t) \nabla(u(\tau)-u(t)) d x d \tau
$$




$$
\begin{aligned}
& +\int_{0}^{t} g_{1}(t-\tau)\|\nabla u(t)\|_{2}^{2} d \tau \\
= & \int_{0}^{t} g_{1}(t-\tau) \int_{\Omega} \nabla u(t)(\nabla u(\tau)-\nabla u(t)) d x d \tau \\
& +\int_{0}^{t} g_{1}(\tau) d \tau\|\nabla u(t)\|_{2}^{2} .
\end{aligned}
$$

In the same way, we have

$$
\begin{aligned}
\int_{0}^{t} g_{2}(t-\tau) \int_{\Omega} \nabla v(\tau) \nabla v(t) d x d \tau= & \int_{0}^{t} g_{2}(t-\tau) \int_{\Omega} \nabla v(t)(\nabla v(\tau)-\nabla v(t)) d x d \tau \\
& +\int_{0}^{t} g_{2}(\tau) d \tau\|\nabla v(t)\|_{2}^{2} .
\end{aligned}
$$

Then, inserting (3.7) and (3.8) into (3.5) and (3.6), respectively,

$$
\begin{aligned}
\frac{d}{d t}\left(u, \frac{u_{t}\left|u_{t}\right|^{\rho}}{\rho+1}\right) & \\
= & \frac{1}{\rho+1}\left\|u_{t}(t)\right\|_{\rho+2}^{\rho+2}-\|\nabla u\|_{2}^{2}+\int_{0}^{t} g_{1}(t-\tau) \int_{\Omega} \nabla u(t)(\nabla u(\tau)-\nabla u(t)) d x d \tau \\
& \quad+\int_{0}^{t} g_{1}(\tau) d \tau\|\nabla u(t)\|_{2}^{2}-\int_{\Omega} u u_{t}\left|u_{t}\right|^{m-2} d x+\int_{\Omega} u f_{1}(u, v) d x, \\
\frac{d}{d t}\left(v, \frac{v_{t}\left|v_{t}\right|^{\rho}}{\rho+1}\right) & \\
= & \frac{1}{\rho+1}\left\|v_{t}(t)\right\|_{\rho+2}^{\rho+2}-\|\nabla v\|_{2}^{2}+\int_{0}^{t} g_{2}(t-\tau) \int_{\Omega} \nabla v(t)(\nabla v(\tau)-\nabla v(t)) d x d \tau \\
& +\int_{0}^{t} g_{2}(\tau) d \tau\|\nabla v(t)\|_{2}^{2}-\int_{\Omega} v v_{t}\left|v_{t}\right|^{r-2} d x+\int_{\Omega} v f_{2}(u, v) d x .
\end{aligned}
$$

For the third term on the right side of (3.9), applying the Cauchy inequality, we obtain

$$
\begin{aligned}
& \int_{0}^{t} g_{1}(t-\tau) \int_{\Omega} \nabla u(t)(\nabla u(\tau)-\nabla u(t)) d x d \tau \\
& \quad \geq-\frac{2(p+2)(1-\varepsilon)}{2}\left(g_{1} \circ \nabla u\right)(t)-\frac{1}{4(p+2)(1-\varepsilon)} \int_{0}^{t} g_{1}(\tau) d \tau\|\nabla u\|_{2}^{2}
\end{aligned}
$$

where $\varepsilon \in(0,1)$, similarly

$$
\begin{aligned}
& \int_{0}^{t} g_{2}(t-\tau) \int_{\Omega} \nabla v(t)(\nabla v(\tau)-\nabla v(t)) d x d \tau \\
& \quad \geq-\frac{2(p+2)(1-\varepsilon)}{2}\left(g_{2} \circ \nabla v\right)(t)-\frac{1}{4(p+2)(1-\varepsilon)} \int_{0}^{t} g_{2}(\tau) d \tau\|\nabla v\|_{2}^{2} .
\end{aligned}
$$

Inserting (3.11) and (3.12) into (3.9) and (3.10), respectively, we can get

$$
\frac{d}{d t}\left(u, \frac{u_{t}\left|u_{t}\right|^{\rho}}{\rho+1}\right) \geq \frac{1}{\rho+1}\left\|u_{t}(t)\right\|_{\rho+2}^{\rho+2}-\|\nabla u\|_{2}^{2}-\frac{2(p+2)(1-\varepsilon)}{2}\left(g_{1} \circ \nabla u\right)(t)
$$




$$
\begin{aligned}
& +\int_{0}^{t} g_{1}(\tau) d \tau\|\nabla u(t)\|_{2}^{2}-\int_{\Omega} u u_{t}\left|u_{t}\right|^{m-2} d x+\int_{\Omega} u f_{1}(u, v) d x \\
& -\frac{1}{4(p+2)(1-\varepsilon)} \int_{0}^{t} g_{1}(\tau) d \tau\|\nabla u\|_{2}^{2}, \\
\frac{d}{d t}\left(v, \frac{\nu_{t}\left|v_{t}\right|^{\rho}}{\rho+1}\right) \geq & \frac{1}{\rho+1}\left\|v_{t}(t)\right\|_{\rho+2}^{\rho+2}-\|\nabla v\|_{2}^{2}-\frac{2(p+2)(1-\varepsilon)}{2}\left(g_{2} \circ \nabla v\right)(t) \\
& +\int_{0}^{t} g_{2}(\tau) d \tau\|\nabla v(t)\|_{2}^{2}-\int_{\Omega} v v_{t}\left|v_{t}\right|^{r-2} d x+\int_{\Omega} v f_{2}(u, v) d x \\
& -\frac{1}{4(p+2)(1-\varepsilon)} \int_{0}^{t} g_{2}(\tau) d \tau\|\nabla v\|_{2}^{2} .
\end{aligned}
$$

Adding (3.13) and (3.14), we derive that

$$
\begin{aligned}
\frac{d}{d t}\left(u, \frac{u_{t}\left|u_{t}\right|^{\rho}}{\rho+1}\right)+\frac{d}{d t}\left(v, \frac{v_{t}\left|v_{t}\right|^{\rho}}{\rho+1}\right) \\
\geq \frac{1}{\rho+1}\left(\left\|u_{t}(t)\right\|_{\rho+2}^{\rho+2}+\left\|v_{t}(t)\right\|_{\rho+2}^{\rho+2}\right)+2(p+2) \int_{\Omega} F(u, v) d x \\
\quad-\left(1-\int_{0}^{t} g_{1}(\tau) d \tau\right)\|\nabla u\|_{2}^{2}-\left(1-\int_{0}^{t} g_{2}(\tau) d \tau\right)\|\nabla v\|_{2}^{2} \\
\quad-\frac{2(p+2)(1-\varepsilon)}{2}\left(\left(g_{1} \circ \nabla u\right)(t)+\left(g_{2} \circ \nabla v\right)(t)\right) \\
\quad-\frac{1}{4(p+2)(1-\varepsilon)}\left(\int_{0}^{t} g_{1}(\tau) d \tau\|\nabla u\|_{2}^{2}+\int_{0}^{t} g_{2}(\tau) d \tau\|\nabla v\|_{2}^{2}\right) \\
\quad-\int_{\Omega} u u_{t}\left|u_{t}\right|^{m-2} d x-\int_{\Omega} v v_{t}\left|v_{t}\right|^{r-2} d x .
\end{aligned}
$$

Adding $2(p+2)(1-\varepsilon) E(t)$ on the right side of (3.15), we can get

$$
\begin{aligned}
& \frac{d}{d t}\left(u, \frac{u_{t}\left|u_{t}\right|^{\rho}}{\rho+1}\right)+\frac{d}{d t}\left(v, \frac{v_{t}\left|v_{t}\right|^{\rho}}{\rho+1}\right) \\
& \geq\left(\frac{1}{\rho+1}+\frac{2(p+2)(1-\varepsilon)}{\rho+2}\right)\left(\left\|u_{t}(t)\right\|_{\rho+2}^{\rho+2}+\left\|v_{t}(t)\right\|_{\rho+2}^{\rho+2}\right) \\
& \quad+2(p+2) \varepsilon \int_{\Omega} F(u, v)-2(p+2)(1-\varepsilon) E(t) \\
& \quad+((p+2)(1-\varepsilon)-1)\left(1-\int_{0}^{t} g_{1}(\tau) d \tau\right)\|\nabla u\|_{2}^{2} \\
& \quad+((p+2)(1-\varepsilon)-1)\left(1-\int_{0}^{t} g_{2}(\tau) d \tau\right)\|\nabla v\|_{2}^{2} \\
& \quad-\frac{1}{4(p+2)(1-\varepsilon)}\left(\int_{0}^{t} g_{1}(\tau) d \tau\|\nabla u\|_{2}^{2}+\int_{0}^{t} g_{2}(\tau) d \tau\|\nabla v\|_{2}^{2}\right) \\
& \quad-\int_{\Omega} u u_{t}\left|u_{t}\right|^{m-2} d x-\int_{\Omega} v v_{t}\left|v_{t}\right|^{r-2} d x .
\end{aligned}
$$


For the last two terms on the right side of (3.16), applying the Hölder inequality and the Young inequality, we arrive at

$$
\left.\left|\int_{\Omega}\right| u_{t}\right|^{m-2} u_{t} u d x \mid \leq \frac{\varepsilon_{1}^{m}\|u\|_{m}^{m}}{m}+\left(\frac{1}{\varepsilon_{1}}\right)^{\frac{m}{m-1}} \frac{(m-1)\left\|u_{t}\right\|_{m}^{m}}{m},
$$

where $\varepsilon_{1}>0$. By Lemma 2.4 and the conditions of the theorem, one can deduce that $\|u\|_{m}^{m} \leq\|u\|_{2}^{2}+\|u\|_{2(p+2)}^{2(p+2)}$, then the inequality (3.17) can be rewritten as

$$
\left.\left|\int_{\Omega}\right| u_{t}\right|^{m-2} u_{t} u d x \mid \leq \frac{\varepsilon_{1}^{m}}{m}\left(\|u\|_{2}^{2}+\|u\|_{2(p+2)}^{2(p+2)}\right)+\left(\frac{1}{\varepsilon_{1}}\right)^{\frac{m}{m-1}} \frac{(m-1)\left\|u_{t}\right\|_{m}^{m}}{m},
$$

similarly, we have

$$
\left.\left|\int_{\Omega}\right| \nu_{t}\right|^{r-2} v_{t} \nu d x \mid \leq \frac{\varepsilon_{1}^{r}}{r}\left(\|v\|_{2}^{2}+\|v\|_{2(p+2)}^{2(p+2)}\right)+\left(\frac{1}{\varepsilon_{1}}\right)^{\frac{r}{r-1}} \frac{(r-1)\left\|v_{t}\right\|_{r}^{r}}{r} .
$$

Substituting (3.18) and (3.19) into (3.16), then we have

$$
\begin{aligned}
\frac{d}{d t}( & \left.u, \frac{u_{t}\left|u_{t}\right|^{\rho}}{\rho+1}\right)+\frac{d}{d t}\left(v, \frac{v_{t}\left|v_{t}\right|^{\rho}}{\rho+1}\right)+\left(\frac{1}{\varepsilon_{1}}\right)^{\frac{m}{m-1}} \frac{(m-1)\left\|u_{t}\right\|_{m}^{m}}{m}+\left(\frac{1}{\varepsilon_{1}}\right)^{\frac{r}{r-1}} \frac{(r-1)\left\|v_{t}\right\|_{r}^{r}}{r} \\
\geq & \left(\frac{1}{\rho+1}+\frac{2(p+2)(1-\varepsilon)}{\rho+2}\right)\left(\left\|u_{t}(t)\right\|_{\rho+2}^{\rho+2}+\left\|v_{t}(t)\right\|_{\rho+2}^{\rho+2}\right) \\
& +2(p+2) \varepsilon \int_{\Omega} F(u, v)-2(p+2)(1-\varepsilon) E(t) \\
& +((p+2)(1-\varepsilon)-1)\left(1-\int_{0}^{t} g_{1}(\tau) d \tau\right)\|\nabla u\|_{2}^{2} \\
& +((p+2)(1-\varepsilon)-1)\left(1-\int_{0}^{t} g_{2}(\tau) d \tau\right)\|\nabla v\|_{2}^{2} \\
& -\frac{1}{4(p+2)(1-\varepsilon)}\left(\int_{0}^{t} g_{1}(\tau) d \tau\|\nabla u\|_{2}^{2}+\int_{0}^{t} g_{2}(\tau) d \tau\|\nabla v\|_{2}^{2}\right) \\
& -\frac{\varepsilon_{1}^{r}}{r}\left(\|v\|_{2}^{2}+\|v\|_{2(p+2)}^{2(p+2)}\right)-\frac{\varepsilon_{1}^{m}}{m}\left(\|u\|_{2}^{2}+\|u\|_{2(p+2)}^{2(p+2)}\right) .
\end{aligned}
$$

Take $\gamma=\max \{m, r\}, \gamma^{*}=\min \{m, r\}$. Combining with (2.5), we know $E^{\prime}(t) \leq-\left\|u_{t}\right\|_{m}^{m}-\left\|v_{t}\right\|_{r}^{r}$, that is, $-E^{\prime}(t) \geq\left\|u_{t}\right\|_{m}^{m}+\left\|v_{t}\right\|_{r}^{r}$, then (3.20) can be rewritten as

$$
\begin{aligned}
& \frac{d}{d t}\left(\left(u, \frac{u_{t}\left|u_{t}\right|^{\rho}}{\rho+1}\right)+\left(v, \frac{v_{t}\left|v_{t}\right|^{\rho}}{\rho+1}\right)-\left(\frac{1}{\varepsilon_{1}}\right)^{\frac{\gamma^{*}}{\gamma^{*}-1}} \frac{\gamma-1}{\gamma} E(t)\right) \\
& \geq \frac{d}{d t}\left(\left(u, \frac{u_{t}\left|u_{t}\right|^{\rho}}{\rho+1}\right)+\left(v, \frac{v_{t}\left|v_{t}\right|^{\rho}}{\rho+1}\right)\right)+\left(\frac{1}{\varepsilon_{1}}\right)^{\frac{\gamma^{*}}{\gamma^{*-1}}} \frac{\gamma-1}{\gamma}\left(\left\|u_{t}\right\|_{m}^{m}+\left\|v_{t}\right\|_{r}^{r}\right) \\
& \geq \frac{d}{d t}\left(u, \frac{u_{t}\left|u_{t}\right|^{\rho}}{\rho+1}\right)+\frac{d}{d t}\left(v, \frac{v_{t}\left|v_{t}\right|^{\rho}}{\rho+1}\right)+\left(\frac{1}{\varepsilon_{1}}\right)^{\frac{m}{m-1}} \frac{(m-1)\left\|u_{t}\right\|_{m}^{m}}{m} \\
& \quad+\left(\frac{1}{\varepsilon_{1}}\right)^{\frac{r}{r-1}} \frac{(r-1)\left\|v_{t}\right\|_{r}^{r}}{r}
\end{aligned}
$$




$$
\begin{aligned}
\geq & \left(\frac{1}{\rho+1}+\frac{2(p+2)(1-\varepsilon)}{\rho+2}\right)\left(\left\|u_{t}(t)\right\|_{\rho+2}^{\rho+2}+\left\|v_{t}(t)\right\|_{\rho+2}^{\rho+2}\right) \\
& +2(p+2) \varepsilon \int_{\Omega} F(u, v) d x-2(p+2)(1-\varepsilon) E(t) \\
& +((p+2)(1-\varepsilon)-1)\left(1-\int_{0}^{t} g_{1}(\tau) d \tau\right)\|\nabla u\|_{2}^{2} \\
& +((p+2)(1-\varepsilon)-1)\left(1-\int_{0}^{t} g_{2}(\tau) d \tau\right)\|\nabla v\|_{2}^{2} \\
& -\frac{1}{4(p+2)(1-\varepsilon)}\left(\int_{0}^{t} g_{1}(\tau) d \tau\|\nabla u\|_{2}^{2}+\int_{0}^{t} g_{2}(\tau) d \tau\|\nabla v\|_{2}^{2}\right) \\
& -\frac{\varepsilon_{1}^{r}}{r}\left(\|v\|_{2}^{2}+\|v\|_{2(p+2)}^{2(p+2)}\right)-\frac{\varepsilon_{1}^{m}}{m}\left(\|u\|_{2}^{2}+\|u\|_{2(p+2)}^{2(p+2)}\right) .
\end{aligned}
$$

Combining Lemma 2.3 and the Poincaré inequality, we can deduce

$$
\begin{aligned}
\frac{d}{d t}( & \left.\left(u, \frac{u_{t}\left|u_{t}\right|^{\rho}}{\rho+1}\right)+\left(v, \frac{v_{t}\left|v_{t}\right|^{\rho}}{\rho+1}\right)-\left(\frac{1}{\varepsilon_{1}}\right)^{\frac{\gamma^{*}}{\gamma^{*}-1}} \frac{\gamma-1}{\gamma} E(t)\right) \\
\geq & \left(\frac{1}{\rho+1}+\frac{2(p+2)(1-\varepsilon)}{\rho+2}\right)\left(\left\|u_{t}(t)\right\|_{\rho+2}^{\rho+2}+\left\|v_{t}(t)\right\|_{\rho+2}^{\rho+2}\right) \\
& +c_{0} \varepsilon\left(\|u\|_{2(p+2)}^{2(p+2)}+\|v\|_{2(p+2)}^{2(p+2)}\right)-2(p+2)(1-\varepsilon) E(t) \\
& +\left[((p+2)(1-\varepsilon)-1)\left(1-\int_{0}^{t} g_{1}(\tau) d \tau\right)-\frac{1}{4(p+2)(1-\varepsilon)} \int_{0}^{t} g_{1}(\tau) d \tau\right] \lambda\|u\|_{2}^{2} \\
& +\left[((p+2)(1-\varepsilon)-1)\left(1-\int_{0}^{t} g_{2}(\tau) d \tau\right)-\frac{1}{4(p+2)(1-\varepsilon)} \int_{0}^{t} g_{2}(\tau) d \tau\right] \lambda\|v\|_{2}^{2} \\
& -\frac{\varepsilon_{1}^{r}}{r}\left(\|v\|_{2}^{2}+\|v\|_{2(p+2)}^{2(p+2)}\right)-\frac{\varepsilon_{1}^{m}}{m}\left(\|u\|_{2}^{2}+\|u\|_{2(p+2)}^{2(p+2)}\right) \\
\geq & \left(\frac{1}{\rho+1}+\frac{2(p+2)(1-\varepsilon)}{\rho+2}\right)\left(\left\|u_{t}(t)\right\|_{\rho+2}^{\rho+2}+\left\|v_{t}(t)\right\|_{\rho+2}^{\rho+2}\right) \\
& +c_{0} \varepsilon\left(\|u\|_{2(p+2)}^{2(p+2)}+\|v\|_{2(p+2)}^{2(p+2)}\right)-2(p+2)(1-\varepsilon) E(t) \\
& +\left[((p+2)(1-\varepsilon)-1) l_{1}-\frac{1}{4(p+2)(1-\varepsilon)}\left(1-l_{1}\right)\right] \lambda\|u\|_{2}^{2} \\
& +\left[((p+2)(1-\varepsilon)-1) l_{2}-\frac{1}{4(p+2)(1-\varepsilon)}\left(1-l_{2}\right)\right] \lambda\|v\|_{2}^{2} \\
& -\frac{\varepsilon_{1}^{r}}{r}\left(\|v\|_{2}^{2}+\|v\|_{2(p+2)}^{2(p+2)}\right)-\frac{\varepsilon_{1}^{m}}{m}\left(\|u\|_{2}^{2}+\|u\|_{2(p+2)}^{2(p+2)}\right),
\end{aligned}
$$

where $\lambda$ is the first eigenvalue of $-\Delta$, now we take

$$
\begin{aligned}
& \kappa_{1}(\varepsilon)=((p+2)(1-\varepsilon)-1) l_{1}-\frac{1}{4(p+2)(1-\varepsilon)}\left(1-l_{1}\right), \\
& \kappa_{2}(\varepsilon)=((p+2)(1-\varepsilon)-1) l_{2}-\frac{1}{4(p+2)(1-\varepsilon)}\left(1-l_{2}\right) .
\end{aligned}
$$


Then (3.22) can be rewritten as

$$
\begin{aligned}
& \frac{d}{d t}\left(\left(u, \frac{u_{t}\left|u_{t}\right|^{\rho}}{\rho+1}\right)+\left(v, \frac{v_{t}\left|v_{t}\right|^{\rho}}{\rho+1}\right)-\left(\frac{1}{\varepsilon_{1}}\right)^{\frac{\gamma^{*}}{\gamma^{*}-1}} \frac{\gamma-1}{\gamma} E(t)\right) \\
& \geq\left(\frac{1}{\rho+1}+\frac{2(p+2)(1-\varepsilon)}{\rho+2}\right)\left(\left\|u_{t}(t)\right\|_{\rho+2}^{\rho+2}+\left\|v_{t}(t)\right\|_{\rho+2}^{\rho+2}\right) \\
& \quad+\left(\kappa_{1}(\varepsilon) \lambda-\frac{\varepsilon_{1}^{m}}{m}\right)\|u\|_{2}^{2}+\left(\kappa_{2}(\varepsilon) \lambda-\frac{\varepsilon_{1}^{r}}{r}\right)\|v\|_{2}^{2} \\
& \quad+\left(c_{0} \varepsilon-\frac{\varepsilon_{1}^{r}}{r}\right)\|v\|_{2(p+2)}^{2(p+2)}+\left(c_{0} \varepsilon-\frac{\varepsilon_{1}^{m}}{m}\right)\|u\|_{2(p+2)}^{2(p+2)} \\
& \quad-2(p+2)(1-\varepsilon) E(t) .
\end{aligned}
$$

By the condition

$$
\max \left\{\int_{0}^{\infty} g_{1}(\tau) d \tau, \int_{0}^{\infty} g_{2}(\tau) d \tau\right\}<\frac{p+1}{p+1+\frac{1}{4(p+2)}}
$$

we can obtain

$$
\begin{aligned}
& ((p+2)-1) l_{1}-\frac{1}{4(p+2)}\left(1-l_{1}\right)>0 \\
& ((p+2)-1) l_{2}-\frac{1}{4(p+2)}\left(1-l_{2}\right)>0 .
\end{aligned}
$$

Then we choose $\varepsilon$ small enough such that

$$
\begin{aligned}
& \kappa_{1}(\varepsilon)=((p+2)(1-\varepsilon)-1) l_{1}-\frac{1}{4(p+2)(1-\varepsilon)}\left(1-l_{1}\right)>0, \\
& \kappa_{2}(\varepsilon)=((p+2)(1-\varepsilon)-1) l_{2}-\frac{1}{4(p+2)(1-\varepsilon)}\left(1-l_{2}\right)>0 .
\end{aligned}
$$

And we pick $\varepsilon_{1}$ small enough such that

$$
\begin{aligned}
& \min \left\{\kappa_{1}(\varepsilon) \lambda-\frac{\varepsilon_{1}^{m}}{m}, \kappa_{2}(\varepsilon) \lambda-\frac{\varepsilon_{1}^{r}}{r}\right\}>0, \\
& \min \left\{c_{0} \varepsilon-\frac{\varepsilon_{1}^{m}}{m}, c_{0} \varepsilon-\frac{\varepsilon_{1}^{r}}{r}\right\}>0 .
\end{aligned}
$$

Then we choose

$$
\begin{aligned}
& \alpha=\min \left\{\kappa_{1}(\varepsilon) \lambda-\frac{\varepsilon_{1}^{m}}{m}, \kappa_{2}(\varepsilon) \lambda-\frac{\varepsilon_{1}^{r}}{r}, c_{0} \varepsilon-\frac{\varepsilon_{1}^{m}}{m}, c_{0} \varepsilon-\frac{\varepsilon_{1}^{r}}{r}\right\}, \\
& \beta=\min \left\{\frac{1}{\rho+1}+\frac{2(p+2)(1-\varepsilon)}{\rho+2}, \alpha\right\} .
\end{aligned}
$$


Using Lemma 2.4, (3.23) can be deduced as

$$
\begin{aligned}
\frac{d}{d t}( & \left.\left(u, \frac{u_{t}\left|u_{t}\right|^{\rho}}{\rho+1}\right)+\left(v, \frac{v_{t}\left|v_{t}\right|^{\rho}}{\rho+1}\right)-\left(\frac{1}{\varepsilon_{1}}\right)^{\frac{\gamma^{*}}{\gamma^{*}-1}} \frac{\gamma-1}{\gamma} E(t)\right) \\
\geq & \left(\frac{1}{\rho+1}+\frac{2(p+2)(1-\varepsilon)}{\rho+2}\right)\left(\left\|u_{t}(t)\right\|_{\rho+2}^{\rho+2}+\left\|v_{t}(t)\right\|_{\rho+2}^{\rho+2}\right) \\
& +\alpha\left(\|u\|_{2}^{2}+\|u\|_{2(p+2)}^{2(p+2)}\right)+\alpha\left(\|v\|_{2}^{2}+\|v\|_{2(p+2)}^{2(p+2)}\right) \\
& -2(p+2)(1-\varepsilon) E(t) \\
\geq & \beta\left(\left\|u_{t}(t)\right\|_{\rho+2}^{\rho+2}+\left\|v_{t}(t)\right\|_{\rho+2}^{\rho+2}\right) \\
& +\beta\left(\|u\|_{2}^{2}+\|u\|_{2(p+2)}^{2(p+2)}\right)+\beta\left(\|v\|_{2}^{2}+\|v\|_{2(p+2)}^{2(p+2)}\right) \\
& -2(p+2)(1-\varepsilon) E(t) \\
\geq & \beta\left(\left\|u_{t}(t)\right\|_{\rho+2}^{\rho+2}+\left\|v_{t}(t)\right\|_{\rho+2}^{\rho+2}\right)+\beta\|u\|_{\rho+2}^{\rho+2}+\beta\|v\|_{\rho+2}^{\rho+2}-2(p+2)(1-\varepsilon) E(t) .
\end{aligned}
$$

By applying the Hölder and Young inequalities, we can deduce that

$$
\left(u, \frac{u_{t}\left|u_{t}\right|^{\rho}}{\rho+1}\right) \leq\|u\|_{\rho+2}^{\rho+2}+\left\|u_{t}\right\|_{\rho+2}^{\rho+2}
$$

then we have

$$
\begin{aligned}
& \frac{d}{d t}\left(\left(u, \frac{u_{t}\left|u_{t}\right|^{\rho}}{\rho+1}\right)+\left(v, \frac{v_{t}\left|v_{t}\right|^{\rho}}{\rho+1}\right)-\left(\frac{1}{\varepsilon_{1}}\right)^{\frac{\gamma^{*}}{\gamma^{*}-1}} \frac{\gamma-1}{\gamma} E(t)\right) \\
& \quad \geq \beta\left(\left(u, \frac{u_{t}\left|u_{t}\right|^{\rho}}{\rho+1}\right)+\left(v, \frac{v_{t}\left|v_{t}\right|^{\rho}}{\rho+1}\right)-\frac{2(p+2)(1-\varepsilon)}{\beta} E(t)\right)
\end{aligned}
$$

It is easy to see that

$$
\left(\frac{1}{\varepsilon_{1}}\right)^{\frac{\gamma^{*}}{\gamma^{*}-1}} \frac{\gamma-1}{\gamma} \rightarrow+\infty, \quad \varepsilon_{1} \rightarrow 0^{+},
$$

and $\frac{2(p+2)(1-\varepsilon)}{\beta}$ is a positive constant, hence there exists a constant $\varepsilon_{1}^{*}$ such that

$$
\left(\frac{1}{\varepsilon_{1}}\right)^{\frac{\gamma^{*}}{\gamma^{*}-1}} \frac{\gamma-1}{\gamma} \geq \frac{2(p+2)(1-\varepsilon)}{\beta} .
$$

Therefore, we have

$$
\begin{aligned}
& \frac{d}{d t}\left(\left(u, \frac{u_{t}\left|u_{t}\right|^{\rho}}{\rho+1}\right)+\left(v, \frac{v_{t}\left|v_{t}\right|^{\rho}}{\rho+1}\right)-\left(\frac{1}{\varepsilon_{1}^{*}}\right)^{\frac{\gamma^{*}}{\gamma^{*}-1}} \frac{\gamma-1}{\gamma} E(t)\right) \\
& \quad \geq \beta\left(\left(u, \frac{u_{t}\left|u_{t}\right|^{\rho}}{\rho+1}\right)+\left(v, \frac{v_{t}\left|v_{t}\right|^{\rho}}{\rho+1}\right)-\left(\frac{1}{\varepsilon_{1}^{*}}\right)^{\frac{\gamma^{*}}{\gamma^{*}-1}} \frac{\gamma-1}{\gamma} E(t)\right) .
\end{aligned}
$$


Take

$$
H(t)=\left(u, \frac{u_{t}\left|u_{t}\right|^{\rho}}{\rho+1}\right)+\left(v, \frac{v_{t}\left|v_{t}\right|^{\rho}}{\rho+1}\right)-\left(\frac{1}{\varepsilon_{1}^{*}}\right)^{\frac{\gamma^{*}}{\gamma *-1}} \frac{\gamma-1}{\gamma} E(t),
$$

from (3.2), we know

$$
H(0)=\left(u(0), \frac{u_{t}(0)\left|u_{t}(0)\right|^{\rho}}{\rho+1}\right)+\left(v(0), \frac{v_{t}(0)\left|v_{t}(0)\right|^{\rho}}{\rho+1}\right)-\left(\frac{1}{\varepsilon_{1}^{*}}\right)^{\frac{\gamma^{*}}{\gamma^{*-1}}} \frac{\gamma-1}{\gamma} E(0)>0 .
$$

By calculating $H^{\prime}(t) \geq \beta H(t)$, we can get

$$
H(t) \geq e^{\beta t} H(0), \quad \forall t \geq 0 .
$$

Since $(u, v)$ shows global existence, by Lemma 2.2 and Lemma 2.6, we have $0<E(t) \leq$ $E(0), t \in[0,+\infty)$, then

$$
\begin{aligned}
\|u\|_{\rho+2}^{\rho+2}+\left\|u_{t}\right\|_{\rho+2}^{\rho+2}+\|v\|_{\rho+2}^{\rho+2}+\left\|v_{t}\right\|_{\rho+2}^{\rho+2} & \geq\left(u, \frac{u_{t}\left|u_{t}\right|^{\rho}}{\rho+1}\right)+\left(u, \frac{u_{t}\left|u_{t}\right|^{\rho}}{\rho+1}\right) \\
& \geq e^{\beta t} H(0) .
\end{aligned}
$$

Using the Hölder inequality, Lemma 2.2 and Lemma 2.6, we have

$$
\begin{aligned}
\|u(t)\|_{\rho+2}+\|v\|_{\rho+2} \\
\leq\|u(0)\|_{\rho+2}+\|v(0)\|_{\rho+2}+\int_{0}^{t}\left\|u_{t}(\tau)\right\|_{\rho+2} d \tau+\int_{0}^{t}\left\|v_{t}(\tau)\right\|_{\rho+2} d \tau \\
\leq\|u(0)\|_{\rho+2}+\|v(0)\|_{\rho+2}+C_{1} \int_{0}^{t}\left\|u_{t}(\tau)\right\|_{m} d \tau+C_{2} \int_{0}^{t}\left\|v_{t}(\tau)\right\|_{r} d \tau \\
\leq\|u(0)\|_{\rho+2}+\|v(0)\|_{\rho+2}+C_{1} t^{\frac{m-1}{m}}\left(\int_{0}^{t}\left\|u_{t}(\tau)\right\|_{m} d \tau\right)^{\frac{1}{m}} \\
\quad+C_{2} t^{\frac{r-1}{r}}\left(\int_{0}^{t}\left\|v_{t}(\tau)\right\|_{r} d \tau\right)^{\frac{1}{r}} \\
\leq\|u(0)\|_{\rho+2}+\|v(0)\|_{\rho+2}+C_{1} t^{\frac{m-1}{m}}(E(0)-E(t))^{\frac{1}{m}}+C_{2} t^{\frac{r-1}{r}}(E(0)-E(t))^{\frac{1}{r}} \\
\leq\|u(0)\|_{\rho+2}+\|v(0)\|_{\rho+2}+C_{1} t^{\frac{m-1}{m}}(E(0))^{\frac{1}{m}}+C_{2} t^{\frac{r-1}{r}}(E(0))^{\frac{1}{r}},
\end{aligned}
$$

where $C_{1}$ and $C_{2}$ are positive constants. By combining (3.29) and (3.30), we know $\|u\|_{\rho+2}^{\rho+2}+$ $\|v\|_{\rho+2}^{\rho+2}$ shows polynomial growth and $\left\|u_{t}\right\|_{\rho+2}^{\rho+2}+\left\|v_{t}\right\|_{\rho+2}^{\rho+2}$ shows exponential growth. By (2.5) and $E(t)$ being nonnegative, we can deduce

$$
\int_{0}^{t}\left\|u_{t}(\tau)\right\|_{m}^{m} d \tau+\int_{0}^{t}\left\|v_{t}(\tau)\right\|_{r}^{r} d \tau \leq E(0)
$$


and thanks to Lemma 2.5 and the assumption $2<\rho+2<\min \{m, r\}$, we have

$$
\begin{aligned}
& \left\|u_{t}(\tau)\right\|_{m}^{\rho+2}<\left\|u_{t}(\tau)\right\|_{m}^{m}+1, \\
& \left\|v_{t}(\tau)\right\|_{r}^{\rho+2}<\left\|v_{t}(\tau)\right\|_{r}^{r}+1 .
\end{aligned}
$$

By using the Sobolev embedding theorem and combining (3.31) and (3.32), we can get

$$
\begin{aligned}
& \int_{0}^{t}\left\|u_{t}(\tau)\right\|_{\rho+2}^{\rho+2} d \tau+\int_{0}^{t}\left\|v_{t}(\tau)\right\|_{\rho+2}^{\rho+2} d \tau \\
& \quad \leq C\left(\int_{0}^{t}\left\|u_{t}(\tau)\right\|_{m}^{\rho+2} d \tau+\int_{0}^{t}\left\|v_{t}(\tau)\right\|_{r}^{\rho+2} d \tau\right) \\
& \quad \leq C\left(\int_{0}^{t}\left(\left\|u_{t}(\tau)\right\|_{m}^{m}+1\right) d \tau+\int_{0}^{t}\left(\left\|v_{t}(\tau)\right\|_{r}^{r}+1\right) d \tau\right) \\
& \quad \leq C E(0)+2 C t,
\end{aligned}
$$

which contradicts with $\left\|u_{t}\right\|_{\rho+2}^{\rho+2}+\left\|v_{t}\right\|_{\rho+2}^{\rho+2}$ showing exponential growth. Hence the theorem is proved.

\section{Acknowledgements}

The author is very grateful to the referees' valuable suggestions, which improved this work a lot.

Funding

Not applicable.

Availability of data and materials

Data sharing not applicable to this article as no datasets were generated or analyzed during the current study.

\section{Competing interests}

The author declares that they have no competing interests.

\section{Authors' contributions}

The author declares that the author read and approved the final manuscript.

\section{Publisher's Note}

Springer Nature remains neutral with regard to jurisdictional claims in published maps and institutional affiliations.

Received: 6 February 2021 Accepted: 8 June 2021 Published online: 15 July 2021

\section{References}

1. Y.Z. Zhao, Q.P. Wang. Blow-up of arbitrarily positive initial energy solutions for a viscoelastic wave system with nonlinear damping and source terms. Bound. Value Probl., 2018 (2018) 153.

2. Agre, K., Rammaha, M.A.: Systems of nonlinear wave equations with damping and source terms. Differ. Integral Equ. 19(11), 1235-1270 (2006)

3. Cavalcanti, M.M., Domingos Cavalcanti, V.N., Ferreira, J.: Existence and uniform decay for nonlinear viscoelastic equation with strong damping. Math. Mech. Appl. Sci. 24, 1043-1053 (2001)

4. Georgiev, V., Todorova, G.: Existence of a solution of the wave equation with nonlinear damping and source term. J. Differ. Equ. 109, 295-308 (1994)

5. Han, X.S., Wang, M.X.: Global existence and blow up of solutions for a system of nonlinear viscoelastic wave equations with damping and source. Nonlinear Anal. 71, 5427-5450 (2009)

6. Han, X.S., Wang, M.X.: Global existence and blow-up of solutions for a system of nonlinear viscoelastic wave equations with damping and source. Nonlinear Anal. 71, 5427-5450 (2009)

7. Hao, J.H., Wei, H.Y.: Blow-up and global existence for solution of quasilinear viscoelastic wave equation with strong damping and source term. Bound. Value Probl. 1, 65 (2017)

8. He, L.F., Song, H.T.: Global Nonexistence of Arbitrarily Positive Initial Energy Solutions for Viscoelastic Wave Equation. Lanzhou University, Lanzhou (2017)

9. Levien, H.A.: Instability and nonexistence of global solutions of nonlinear wave equation of the form $P u_{t t}=A u+F(u)$. Trans. Am. Math. Soc. 192, 1-21 (1974)

10. Levien, H.A.: Some additional remarks on the nonexistence of global solutions to nonlinear wave equation. SIAM J. Math. Anal. 5, 138-146 (1974) 
11. Messaoudi, S.A.: Blow up and global existence in a nonlinear viscoelastic wave equation. Math. Nachr. 260, 58-66 (2003)

12. Messaoudi, S.A.: Blow up of positive initial energy solution of a nonlinear viscoelastic hyperbolic equation. J. Math Anal. Appl. 320, 902-915 (2006)

13. Messaoudi, S.A., Said-Houari, B.: Global nonexistence of positive initial energy solutions of a system of nonlinear viscoelastic wave equations with damping and source terms. J. Math. Anal. Appl. 365, 277-287 (2010)

14. Mustafa, M.I.: Well posedness and asymptotic behavior of a coupled system of nonlinear viscoelastic equations. Nonlinear Anal., Real World Appl. 13, 452-463 (2012)

15. Papageorgiou, N.S., Radulescu, V.D., Repovs, D.D.: Nonlinear Analysis - Theory and Methods. Springer Monographs in Mathematics. Springer, Cham (2019)

16. Payne, L., Sattinger, D.: Saddle points and instability on nonlinear hyperbolic equation. Isr. J. Math. 22, 273-303 (1975)

17. Said-Houari, B.: Global nonexistence of positive initial-energy solutions of a system of nonlinear wave eqautions with damping and source terms. Differ. Integral Equ. 23(1-2), 79-92 (2010)

18. Song, H.T.: Global nonexistence of positive initial energy solution for a viscoelastic wave. Nonlinear Anal. 125, 260-269 (2015)

19. Song, H.T.: Blow up of arbitrarily positive initial energy solution for a viscoelastic wave euqation. Nonlinear Anal., Real World Appl. 26, 306-314 (2015)

20. Song, H.T., Xue, D.S.: Blow up in a nonlinear viscoelastic wave equation with strong damping. Nonlinear Anal. 109 245-251 (2014)

21. Song, H.T., Zhong, C.K.: Blow up of solution of a nonlinear viscoelastic wave equation. Nonlinear Anal., Real World Appl. 11, 3877-3883 (2010)

22. Xu, R.Z., Lian, W.: Global well-posedness of nonlinear wave equation with weak and strong damping terms and logarithmic source term. Adv. Nonlinear Anal. 9, 613-632 (2020)

23. Xu, R.Z., Lian, W., Niu, Y.: Global well-posedness of coupled parabolic systems. Sci. China Math. 63, 321-356 (2020)

24. Xu, R.Z., Su, J.: Global existence and finite time blow-up for a class of semilinear pseudo-parabolic equations. J. Funct. Anal. 264, 2732-2763 (2013)

\section{Submit your manuscript to a SpringerOpen ${ }^{\circ}$ journal and benefit from:}

- Convenient online submission

- Rigorous peer review

- Open access: articles freely available online

- High visibility within the field

- Retaining the copyright to your article

Submit your next manuscript at $\boldsymbol{\nabla}$ springeropen.com 